\title{
Actitud ante la muerte y conductas de riesgo en alumnos de una universidad pública en el estado de México
}

\author{
Claudia Ivette Moreno Jurado \\ Facultad de Ciencias de la Conducta, \\ Universidad Autónoma del Estado de México, \\ Ciudad de México
}

Recibido: 2 de mayo del 2019 / Aceptado: 15 de mayo del 2019

doi: 10.26439/persona2019.n022(1).4083

El presente estudio tuvo como objetivo conocer la correlación entre la actitud ante la muerte y las conductas de riesgo en alumnos de Psicología del estado de México, utilizando la escala PAM-R (1987), que evalúa actitudes hacia la muerte. Según Morales (2014), la muerte es un proceso natural por el que todo ser vivo tendrá que pasar $y$, por tanto, el simple hecho de pensar en ello puede causar miedo. También se aplicó el cuestionario Youth Risk Behavior Surrey (YRBS) (2011), que evalúa las conductas de riesgo. Según Le Bretón (2012), en estas conductas el individuo juega con la idea de muerte, desafía el miedo y se convence a sí mismo de que siempre tendrá una puerta de salida, independientemente de las condiciones en que se encuentre.

actitud ante la muerte / conductas de riesgo / adolescentes

\section{Attitudes Towards Death and Risk Behaviors in Students of a Public University in the State of Mexico}

This study aimed at establishing the correlation between attitudes towards death and risk behaviors in psychology students of the State of Mexico. To this end, we used the PAM-R scale (1987) which evaluates attitudes towards death. According to Morales (2014), death is a natural process which every living being will have to experience and, therefore, just thinking about death may cause fear. We also used the YRBS questionnaire (2011) that evaluates risk behaviors. As stated by Le Breton (2012), the idea of death is present in risk behaviors, because individuals defy fear and convince themselves that they will always have an exit door, independently of the particular conditions they may deal with.

death attitude / risk behaviors / adolescents

correo electrónico: claudiaivette1145978@hotmail.com 


\section{INTRODUCCIÓN}

Según Morales (2014), la muerte es un proceso natural por el que los seres vivos tendrán que pasar. Para algunas personas, el hecho de pensar en ella puede causar miedo, ansiedad y angustia. Este es el destino más cierto del ser humano y para aceptarlo uno debe ser lo suficientemente maduro. Se puede decir que la mayoría de las personas tienen más miedo al proceso de morir, el deterioro físico y lo dolorosa que puede llegar a ser la enfermedad.

Cuando se desconoce la forma de muerte, pueden manifestarse inquietud y desesperanza, lo cual es contrario a cuando se padece alguna enfermedad terminal, en cuyo caso la aceptación se da más pronto.

La palabra muerte alude a una realidad desconocida. Hablar de ella refiere a algo que es exclusivo de los seres humanos. Para nosotros, es lo que acontece a las demás personas, es decir, morir es siempre lo que le ocurre al otro. Pero, de cierta forma, la esperamos, porque cuando pensamos en la palabra muerte lo primero que nos llega a la cabeza es nuestro propio final (Garza, 2017).

Según Kübler-Ross (1969), la muerte no es aceptable para el propio inconsciente; existe una atemporalidad, lo que significa que el inconsciente es como un niño que no conoce ni el inicio ni el fin. Sin embargo, cuando muere alguien cercano, se hace consciente. En ese momento, se produce un alivio $\mathrm{y}$, asimismo, culpa de sentirse bien por no haber sido uno mismo quien acaba de morir.

Los jóvenes tienen menos conciencia de la realidad de la muerte, no se hacen preguntas sobre ella ni piensan en su propio final. A diferencia de ellos, los adultos mayores la perciben como un hecho cercano, piensan en cómo será cuando llegue y la ven como algo natural de la vida. Esto ocurre porque a lo largo de su existencia han vivido y enfrentado un mayor número de pérdidas físicas, sociales y psicológicas (Uribe, Valderrama y López, 2007).

En las conductas de riesgo se juega con la idea de muerte. De esta forma, el joven muestra un sentimiento de libertad al desafiar el miedo y convencerse a sí mismo de que siempre tendrá una puerta de salida, pase lo que pase. Así, la muerte entra en el campo de su propia batalla y deja de ser una fuerza de destrucción. En este periodo de la vida, el cuerpo se convierte en el campo de batalla de la propia identidad (Le Bretón, 2012). Aunado a esto, se puede decir que las conductas de riesgo se manifiestan de manera frecuente al no poder percatarse uno de los peligros que pueden presentarse con estas.

Concurren varias razones para poner en peligro la vida, dichas razones son conflictivas. Un joven y su historia personal tienen la respuesta del sentido que lleva a cometer el acto; mientras que otro joven, cuando vive la muerte de cerca, se siente satisfecho con su vida y puede llegar a tomar conductas diferentes. Las conductas de riesgo pueden tener su origen en el abandono, la indiferencia familiar o, por el contrario, en la sobreprotección; muchas veces, se aduce la descalificación del padre. Siempre está presente la falta de orientación, el sentimiento de ausencia de límites o de que se impusieron de manera incorrecta (Le Breton, 2012).

A ello contribuyen ciertas características de la juventud, entre las que están la sensación de invulnerabilidad o mortalidad 
negada. Las conductas de riesgo se definen como actuaciones situadas fuera de determinados límites que pueden afectar el desarrollo psicosocial durante la infancia o la adolescencia y tener repercusiones perjudiciales para el futuro. En muchas ocasiones, los jóvenes no tienen experiencia y surgen conductas destructivas en las que se desconocen los riesgos a corto $\mathrm{y}$ largo plazo (Le Bretón, 2012).

Cuando los jóvenes experimentan sentimientos de tristeza o soledad, y no encuentran una salida saludable para sentirse mejor o no tienen a alguna persona con quien puedan hablarlo, suelen recurrir a sustancias químicas en busca del consuelo que no obtienen. Dependiendo de lo que usen, pueden llegar a sentir inconscientemente felicidad, mucha energía y confianza. Cuando se les da la oportunidad de tomar algo que los haga sentir mejor de cierta forma, no pueden resistir y lo consumen (Partnership for Doug-Free Kids, 2017).

Una persona, ya sea joven o no, que ha sufrido mucho y siente malestar puede ver las drogas como una salida, una solución a estos problemas, pues los seres humanos necesitamos sentir placer para enfrentar de una mejor forma la vida (Corona y Peralta, 2011).

Según, estos autores, las conductas de riesgo que más se practican en esta etapa son las violentas y las asociadas a accidentes. Por ello, la primera causa de muerte en jóvenes son los accidentes vehiculares, seguidos por tumores y suicidio.

Asimismo, la actividad sexual sin protección puede llevar a contraer enfermedades. Algunas de sus principales consecuencias son el embarazo no deseado y las enfermedades de transmisión sexual. Al respecto, se destacan los siguientes hallazgos:

- Cada año mueren más de 1,8 millones de jóvenes de 15 a 24 años por causas prevenibles.

- Cada año dan a luz aproximadamente 16 millones de mujeres adolescentes de 15 a 19 años.

- Los jóvenes de 15 a 24 años representaron el $40 \%$ de todos los casos nuevos de infección por $\mathrm{VIH}$ registrados entre los adultos en el 2008.

- En el periodo de un año, aproximadamente $20 \%$ de los adolescentes sufren un problema de salud mental, como depresión o ansiedad.

- Se estima que unos 150 millones de jóvenes consumen tabaco.

- Cada día mueren cerca de 565 jóvenes de 10 a 29 años a causa de la violencia interpersonal.

- Se estima que los traumatismos causados por el tránsito provocan la muerte de unos 1000 jóvenes cada día (OMS, 2009).

Los jóvenes manifiestan una cierta atracción por lo negativo, lo cual pueden ver como una apropiación de su destino frente a lo que se les impone, esto en relación con sus necesidades de dependencia. Creen que ellos pueden elegir morir por el hecho de no pedir nacer (Le Breton, 2012).

Para hablar sobre la muerte y las conductas de riesgo, es necesario destacar la actitud ante ello; por tanto, hay que definir esta variable para comprenderla un poco más. Según (González, 2013), y de acuerdo 
con la Real Academia Española, actitud es un término que proviene del latín actitud. Es la disposición de un ánimo que se manifiesta de algún modo, un estado de disposición nerviosa y mental organizado mediante la experiencia, y que ejerce un influjo dinámico u orientador sobre las respuestas que un individuo ofrece a los objetos y las situaciones.

Las actitudes consisten en un componente cognitivo que se traduce en ideas y creencias, relacionadas con el componente afectivo que son las emociones; los dos componentes se vinculan con los componentes conductual, respecto de la actitud. Es decir, la actitud es una disposición de ánimo voluntaria que relaciona el componente cognitivo, afectivo y conductual (González, 2013).

Las actitudes que adoptan las personas ante la muerte suelen presentarse como aceptación y conflicto. Por ello, en el proceso tanatológico los sujetos deben tomar conciencia de las actitudes que manifiestan y cómo influyen en su vida diaria; de esta forma, podrán recibir el apoyo necesario para mejorar su calidad de vida (Morales, 2014).

Un estudio acerca de las conductas de riesgo, efectuado por Huitrón et al. (2011) en la Universidad Autónoma del Estado de México, demuestra que en las últimas décadas se han producido cambios socioculturales demográficos y económicos que han tenido influencia en la estructura y funcionamiento familiar, lo cual se ha visto asociado a modificaciones en los patrones de morbimortalidad de los adolescentes. En la actualidad, múltiples conductas de riesgo de los adolescentes se han vinculado a una serie de problemas de salud pública.

Los resultados obtenidos por Huitrón et al. (2011) sugieren que el funcionamiento familiar es muy importante para la adopción de múltiples conductas de riesgo. Esto quizá por la falta de conocimiento de los padres de las actividades de los hijos y la ausencia o falta de límites en casa. La disminución de la participación de los padres en el proceso educativo de sus hijos y la menor disponibilidad de tiempo son factores que pueden influir para que haya dificultades en la comunicación y una conexión adecuada con los hijos.

También se encontró que tienen mayor riesgo de consumir tabaco, marihuana y tranquilizantes quienes perciben a sus familias como disfuncionales. Asimismo, los padres que consumen tabaco, alcohol u otras sustancias se convierten en un modelo para los hijos. Esto significa que existe un mayor riesgo de que ellos los consuman también.

Tales resultados tienen relación con lo reportado por Blum (en Huitrón et al. 2011), quien menciona que el ambiente familiar y los padres son modelos a seguir, y son vitales para adquirir conductas menos riesgosas, así como el desarrollo sano de todos sus integrantes. Ellos los ayudan a obtener mejores habilidades, más tolerancia y mayor responsabilidad.

En otro estudio, de Bahamón, Vianchá y Tobos (2014), se establece que un alto porcentaje de jóvenes muestran comportamientos de riesgo en cuanto a las relaciones sexuales, lo cual puede causarles problemas con la salud reproductiva, un mayor número de embarazos en adolescentes, 
abortos y enfermedades de transmisión sexual. Se ha encontrado que en los hombres las conductas y las prácticas sexuales se presentan como una forma de reconocimiento sexual, tienen menor riesgo que las mujeres y muestran mayor importancia a lo que dice la sociedad.

En contraposición, las mujeres consideran la sexualidad como un medio para experimentar intimidad y su vinculación emocional se manifiesta con mayor arraigo como un factor para la ejecución de conductas y prácticas de riesgo.

Debido a la presión social, es importante que se les recuerde a los jóvenes que son autónomos y son libres de tomar sus propias decisiones.

En cuanto a la actitud ante la muerte, un estudio realizado por Uribe et al. (2007) muestra que el hecho de pensar en las posibilidades ante la muerte propia o ajena conduce a desplegar una serie de actitudes que puedan ser causa de determinados comportamientos en las personas.

Comúnmente, se ve a la muerte como algo que atenta contra la propia supervivencia, por lo que se responde a ella con temor, que es la actitud más típica. También, produce ansiedad y preocupación, las que motivan en la persona conductas como la evitación de la muerte, aceptación como parte natural de la vida, aceptación de acercamiento (por medio de la cual se concibe la creencia de una vida feliz después de la muerte y se ve como alternativa frente a una vida dolorosa).

Después del estudio realizado por Uribe et al. (2007), se llevó a cabo otro por
Álvarez y Flores (2008). En él se obtiene como respuesta que los adultos intermedios y mayores presentan más aceptación de acercamiento. Esto indica que ven tal acontecimiento como la transición hacia una vida feliz, mantienen una idea integradora acerca de sus vidas y creen haber concluido situaciones pendientes consigo mismos, con los demás y con la vida. Como consecuencia, su percepción de lo vivido es plena y esto hace que su aceptación del final de ese proceso sea esperado con agrado.

Un estudio realizado por Colell, Limonero y Otero (2003) concluye que el aspecto que causa mayor ansiedad está ligado a la propia muerte. Los jóvenes que tienen miedo ante su propia muerte también experimentan mayor ansiedad ante el proceso de su muerte y de la ajena. Sus resultados indicaron que para una población más joven, será más fácil imaginarse la muerte de un ser querido que su propio proceso de morir. Esto implica que tendrán más conocimiento de las enfermedades y mayores experiencias para cuando llegue a ellos.

Las relaciones afectivas pueden desempeñar un papel muy importante, ya que estar cerca de los seres queridos, poder hablar con ellos y pensar que la vida ha tenido un buen sentido ayudará a que las personas puedan morir en paz.

Cada persona tiene diversas necesidades, características y prioridades sobre el proceso de morir, lo cual significa que no hay una forma exacta de describir cuál sería una buena muerte. 


\section{Método}

\section{Participantes}

En esta investigación participaron 201 alumnos de Psicología de una universidad pública del estado de México, los cuales son jóvenes de entre 18 y 25 años de edad.

La muestra se obtuvo mediante un muestreo aleatorio simple con los siguientes criterios de inclusión: ser jóvenes de entre 18 y 25 años de edad, ser estudiantes de la licenciatura en Psicología.

\section{Instrumentos}

- La escala PAM-R se utilizó para evaluar las actitudes hacia la muerte. La crearon Wong, Reker y Gesser (1994) y fue validada por Morales (2014). Contiene afirmaciones relacionadas con diferentes tipos de actitudes en cuanto a aceptación de acercamiento, miedo a la muerte, evitación de la muerte, aceptación de escape, aceptación neutral. Tiene un escalamiento tipo Likert con siete opciones de respuesta, las que van desde totalmente en desacuerdo (1) a totalmente de acuerdo (7). En sus subescalas tiene coeficientes de confiabilidad adecuados: miedo $(\alpha=.86)$, evitación $(\alpha=.88)$, neutral $(\alpha=.65)$, acercamiento $(\alpha=0,97)$, escape $(\alpha=.84)$.

- Es un cuestionario que consta de 32 ítems y el alfa de Cronbach es de 89 . Permite conocer cuáles son las actitudes personales ante la muerte para poder favorecer el enfrentamiento de esta crisis, manejándola de forma abierta y natural como un hecho inevitable que es parte del mismo proceso de la vida.

- El miedo a la muerte se refiere específicamente a la respuesta que tiene el ser humano ante la muerte, quien lo vincula con el temor a la pérdida de sí mismo, al dolor, al sufrimiento.

- La evitación ante la muerte se relaciona con la actitud de rechazo ante el tema de la muerte, como son la falta de diálogo y alejarse de los lugares que se asocien con ella.

- La aceptación neutral es la ambivalencia presente ante el tema de la muerte. Por una parte es un temor normal a la muerte; por otra, la aceptación ante su posible llegada.

- La aceptación de acercamiento se relaciona con creencias religiosas, en las que se tiene una mayor confianza y se piensa en la vida después de ella.

- La aceptación de escape refiere a que la muerte se puede convertir en una alternativa y puede ofrecer el único escape ante emociones negativas.

- El cuestionario sobre conductas de riesgo en adolescentes YRBS, elaborado por el Centro de Control y Prevención de Enfermedades en Atlanta, se usó para medir conductas de riesgo y fue empleado por Burgos (2011). Se ha reportado evidencia de su confiabilidad (Brener et al., 2002; Brener, Collins, Kann, Warren y Williams, 1995) y de validez (Brener, Billy y Grady, 2003).

El instrumento se aplicó a una muestra de estudiantes en dos ocasiones para evaluar su confiabilidad por estabilidad o test-retest y se calculó el coeficiente kappa de Cohen. Se obtuvieron los siguientes resultados e interpretaciones (Landis y Koch, 1977, como se citó en 
Hidalgo, Ramírez, Rojas, Hidalgo y Skalicky, 2017): para los ítems de accidentes, el valor del coeficiente kappa fue de discreto (.26) a sustancial (.73); para los ítems de uso de sustancias, fue de sustancial (.67) a casi perfecto (.94); para violencia y suicidio, fue de discreto (.35) a moderado (.49); para los ítems de riesgo alimentario, fue de sustancial (.66) a casi perfecto (1.0); para los de inactividad física, fue de moderado (.52) a sustancial (.74); y para los de riesgo sexual, fue de discreto (.30) a casi perfecto (1.0).

El Centro de Control y Prevención de Enfermedades en Atlanta desarrolla las preguntas que componen el cuestionario y mide la prevalencia de los comportamientos de riesgo o no-saludables en los adolescentes que son estudiantes del nivel superior en cada estado de Estados Unidos y los territorios. La encuesta trabaja una serie de categorías de conductas de riesgo que son accidentes intencionales y no-intencionales (seguridad personal), uso de cigarrillos (tabaco), uso de alcohol y drogas, comportamiento sexual, comportamiento alimentario y falta de actividad física.

\section{Procedimiento}

Los datos para la correlación entre actitud ante la muerte y conductas de riesgo se obtuvieron mediante dos instrumentos: un cuestionario que mide conductas de riesgo, de fácil comprensión e integrado por preguntas cerradas; y la escala PAM-R para evaluar las actitudes ante la muerte, que es de fácil comprensión y mide distintas actitudes que se tienen ante dicho fenómeno.
Los instrumentos se aplicaron de manera grupal en una universidad del estado de México. Se pidió permiso a los profesores para poder aplicarlos en su grupo; de igual manera, a cada participante se le pidió su colaboración y se entregó una carta de consentimiento informado. La participación fue de manera voluntaria. Para la interpretación de los resultados, se utilizó el programa estadístico SPSS.

\section{Resultados}

En la tabla 1 se muestran las correlaciones entre las actitudes ante la muerte (aceptación, evitación, escape, miedo, neutral) y las conductas de riesgo (seguridad personal, violencia, suicidio, fumar cigarrillos, beber alcohol, consumo de marihuana, de drogas, comportamiento sexual, peso corporal y haber comido o bebido en los últimos 7 días). En este, un asterisco (*) significa que existe una correlación débil; y dos asteriscos $(* *)$, una correlación fuerte.

En el presente trabajo de investigación, que habla sobre la actitud ante la muerte y conductas de riesgo en jóvenes, los resultados indican lo siguiente:

Entre violencia, aceptación (-.144*, $.041)$, escape $\left(-.171^{*}, .015\right)$ y miedo $\left(-.181^{*}\right.$, .010) se da una correlación negativa débil significativa.

Entre fumar cigarrillos y la aceptación $\left(-.181^{* *}, .010\right)$ hay una correlación negativa fuerte significativa; mientras que entre fumar cigarrillos y evitación (-.181*, .010) se da una correlación negativa débil significativa. 
Tabla 1

Matriz de correlación entre actitud ante la muerte y conductas de riesgo

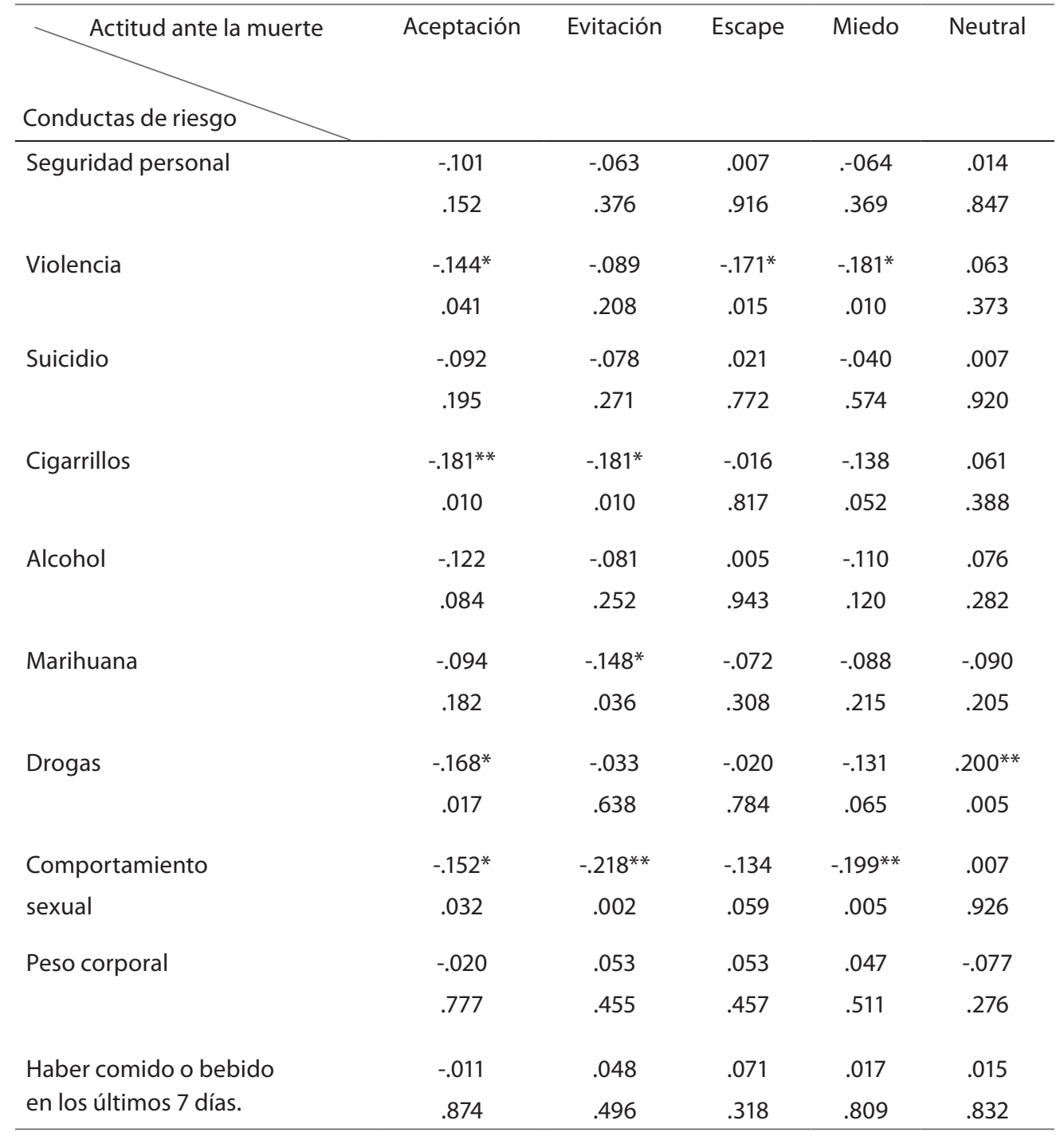

Entre el uso de marihuana y la evitación $\left(-.148^{*}, .036\right)$ hay una correlación negativa débil significativa.
Entre el uso de drogas y la aceptación $(-.168 *, .017)$ se muestra una correlación negativa débil significativa; por otra parte, 
la misma variable, pero en relación con neutral $(.200 * *, .005)$, muestra una correlación positiva fuerte significativa.

El comportamiento sexual y la aceptación $(-.152 *,-032)$ muestran una correlación negativa débil significativa. Por otra parte, en relación con la evitación $(-.218 * *, .002)$ y el miedo $(-.199 * *, .005)$, hay una correlación negativa fuerte y significativa.

\section{DISCUSIÓN}

Esta investigación se desarrolló para analizar las actitudes ante la muerte en relación con las conductas de riesgo en jóvenes. A continuación, se dan a conocer los resultados más significativos: entre violencia, aceptación de acercamiento, aceptación de escape y miedo ante la muerte; entre fumar cigarrillos, aceptación de acercamiento y evitación; entre uso de marihuana y evitación; entre consumo de drogas, aceptación de acercamiento y aceptación neutral; entre comportamiento sexual, aceptación de acercamiento, evitación y miedo ante la muerte.

Según los resultados, entre violencia y aceptación de acercamiento hay una correlación negativa significativa. Para Burgos (2011), la violencia es una característica profundamente persistente que conlleva a consecuencias negativas para el adolescente, su entorno y la sociedad. Por otra parte, la aceptación de acercamiento (actitud ante la muerte) se relaciona con creencias religiosas, en las que se tiene una mayor confianza y está presente la idea de una vida feliz después de la muerte (Morales, 2014).
En la misma variable de violencia se encuentra una correlación negativa significativa con aceptación de escape y miedo ante la muerte. Según Le Bretón (2012), el joven agudiza su sentimiento de libertad, desafía el miedo haciéndole frente y se convence de que todo el tiempo tiene una puerta de salida. En cuanto a la aceptación de escape, el mismo autor menciona que las personas carecen de habilidades de afrontamiento eficaz para enfrentar el dolor que ocasionan los problemas, de modo que la muerte se convierte en una alternativa y puede ofrecer el único escape ante las emociones negativas.

De acuerdo con eso, las personas del estudio que ejercen la violencia, encuentran que en la muerte hay menor escape a las emociones negativas. Quiere decir que enfrentan los problemas de una manera correcta y no ven la muerte como la solución a los problemas. Además, no tienen presente la creencia de una vida después de la muerte, pues no se basan en creencias religiosas. Al ejercer la violencia, se sabe que hay un peligro incluso para las propias personas que la generan, quienes no muestran temor a la pérdida de sí mismas.

En cuanto a la variable de fumar cigarrillos, se menciona que las personas que lo hacen buscan sentirse bien, relajadas o alegres (Burgos, 2011). Se encuentra una correlación negativa significativa con la aceptación de acercamiento y la evitación ante la muerte. La evitación manifiesta una actitud de rechazo ante la muerte y todo lo que se asocie a ella. Esto indica en el presente estudio que las personas que fuman cigarrillos no tienen una creencia de una 
vida feliz después de la muerte, no evitan el tema y dialogan acerca de ello. Por esto, pueden hablar sin temor sobre la muerte y pensar que les puede pasar, pero que falta mucho tiempo para que el cigarrillo les pueda afectar.

El consumo de marihuana se da de manera frecuente, dado que hay facilidad de conseguirla. Al respecto, hay una correlación negativa significativa con la evitación ante la muerte; quiere decir que en el estudio las personas que fuman marihuana la consiguen de forma fácil y no manifiestan rechazo ante el tema de la muerte. En Partnership for Droug-Free Kids (2017) se menciona que algunos de los amigos se presionan entre sí para probar bebidas o fumar algo, pero es igualmente común que los adolescentes empiecen a usar alguna sustancia porque se encuentra fácilmente disponible, además, ven a todos sus amigos disfrutándola. En sus mentes consideran el uso de drogas como una parte normal de la experiencia de ser adolescente $\mathrm{y}$ en muchas ocasiones pueden observar que sus padres o abuelos las utilizan. Esto les da la pauta para pensar que no hacen daño y que si afectan no será a una edad tan temprana, lo cual les facilita el poder hablar de la muerte.

El consumo de drogas muestra una correlación negativa significativa con la aceptación de acercamiento (actitud ante la muerte). En cuanto a la aceptación neutral, hay una correlación positiva significativa. Según Burgos (2011), en el consumo de drogas se encuentra alivio temporal a los problemas que pueden surgir, pero esto afecta su salud física, mental, y amenaza su vida. Aunado a ello, la aceptación neutral indica expresar un temor normal ante la muerte y aceptarla como un hecho innegable en la vida. Dentro de este estudio, quiere decir que las personas consumidoras de drogas encuentran alivio a sus problemas, intentan aprovechar al máximo su vida, ven la muerte como un acontecimiento natural y tienen la creencia de una vida después de ella.

De acuerdo con la investigación de Tejedor (2018), cuando una persona joven o no lleva mucho tiempo sufriendo y sintiendo malestar, las drogas se anuncian como una forma de terminar con eso y de hacerla sentir alegre.

Sobre el comportamiento sexual, se da una correlación negativa fuerte significativa con evitación y miedo a la muerte. Al respecto, según Le Bretón (2012), el joven agudiza su sentimiento de libertad, desafía el miedo al hacerle frente y convencerse de que todo el tiempo tiene una puerta de salida. También se muestra una correlación negativa significativa con la aceptación de acercamiento (actitud ante la muerte), lo cual significa que entre mayor conocimiento tenga el joven sobre sexualidad habrá menor aceptación de acercamiento. No cree en una vida después de la muerte, no agudiza su sentimiento de libertad y no evita dialogar acerca de la muerte.

Burgos (2011) menciona que los jóvenes con vida sexual activa tienen conocimientos suficientes sobre sexualidad. Aun así, se presenta un bajo consumo de anticonceptivos y en el riesgo en las relaciones sexuales.

Las investigaciones señalan que los jóvenes practican conductas de riesgo de 
manera frecuente al no poder percatarse de los peligros que pueden correr. Con relación a ello, contribuyen diferentes características propias de la edad, entre las que destacan la sensación de invulnerabilidad o la mortalidad negada.

Los jóvenes juegan con las conductas de riesgo y la idea de una muerte voluntaria, lo cual quiere decir que hay una puerta de salida en todo lo que surja. La muerte deja de ser una fuerza de destrucción y se convierte en una forma de escape, en una alternativa de solución a los problemas.

En la mayoría de las variables se presentó una correlación negativa débil significativa, a partir de la cual se llega a la conclusión de que la población con que se trabajó tiene creencias religiosas de una vida feliz después de la muerte. Aunque evitan hablar de ella y de todo lo que se relacione con este tema, ven la muerte como una forma de afrontar los problemas que se les pueden presentar y la aceptan como un hecho innegable de la vida. No desarrollan actividades que lleven a consecuencias en las que se arriesguen a sí mismos y a los de su entorno, fuman para sentirse bien, relajados o alegres, y tienen conocimientos suficientes sobre sexualidad $\mathrm{y}$ anticonceptivos.
De acuerdo con esto, se establece una relación significativa con lo que dicen los autores Burgos (2011), Partnership for Droug-Free Kids (2017) y Tejedor (2018). Algunas conductas de riesgo son llevadas a cabo por los jóvenes porque piensan que la muerte es lejana para ellos; llegan a adoptarla como una solución de escape a los problemas y, por lo mismo, la toman como un hecho innegable de la vida.

\section{Comentarios}

Esta investigación proporciona datos que servirán de base para el fomento de programas de prevención, promoción $\mathrm{y}$ protección de la salud en los jóvenes, y en general de la sociedad. Debido a la forma como los jóvenes presentan conductas de riesgo y la noción de muerte voluntaria, es que, de acuerdo con dichos resultados, se requiere el fomento de programas de prevención. Así los jóvenes dejarán de ver la muerte como un escape, ya que algunas conductas de riesgo son llevadas a cabo por ellos porque piensan que la muerte es lejana. Por lo mismo, la tomarán como un hecho innegable de la vida y practicarán de manera menos frecuente dichas conductas de riesgo.

\section{ReFERENCIAS}

Álvarez, Y., y Flores, Z. (2008). Actitudes hacia la muerte en un grupo de adultos intermedios y mayores con enfermedad crónica (hipertensión, diabetes y cáncer) en la ciudad de Bucaramanga. Revista Colombiana de Psicología, 17, 80-81. Recuperado de http:// www.redalyc.org/articulo.oa?id=80411803005

Bahamón, M., Vianchá, M., y Tobos A. (2014) Prácticas y conductas sexuales de riesgo en jóvenes: una perspectiva de género. Psicología desde el Caribe, 2, 342-343. Recuperado de http://www.redalyc.org/articulo.oa?id=21331836009 
Brener, N. D., Kann, L., McManus, T., Kinchen, S., Sundberg, E. C., y Ross, J. (2002). Reliability of the 1999 Youth Risk Behavior Survey Questionnaire. Journal of Adolescent Health, 36, 336-342.

Brener, N. D., Collins, J. L., Kann, L., Warren, C.W., y Williams, B. I. (Mar, 1995). Reliability of the Youth Risk Behavior Survey Questionnaire. American Journal of Epidemiology, 141(6),575-580.

Brener, N. D., Billy, J. O., y Grady, W. R. (2003). Assessment of Factors Affecting the Validity of Self-Reported Health-Risk Behavior Among Adolescents: Evidence From the Scientific Literature. Journal of Adolescent Health, 33(6), 436-457.

Burgos, W. (2011). Encuesta de conductas de riesgo en adolescentes de 9no a 12mo. grado. Youth Risk Behavioral Survey. Recuperado de http://www.estadisticas.gobierno.pr/ iepr/LinkClick.aspx?fileticket $=$ eI0XuiOqeTc\%3d\&tabid=186

Colell, R., Limonero, J., y Otero, M. (2003). Actitudes y emociones en estudiantes de enfermería ante la muerte y la enfermedad terminal. Investigación en Salud, 2, 7-8. Recuperado de http://www.redalyc.org/articulo.oa?id=14250205

Corona, F., y Peralta, E. (2011) Prevención de conductas de riesgo. Rev. Med. Clín. Condes, 22(1), 68-75. Recuperado de https://www.clinicalascondes.cl/dev_clc/media/ imagenes/pdf\%20revista\%20m\%c3\%a9dica/2011/1\%20enero/8_dra_corona-10.pdf

Garza, A. (2017). La muerte del otro. Andamios, 33, 15-18. Recuperado de http://www. redalyc.org/articulo.oa? $\mathrm{id}=62849641002$

González, T. (2013). Actitudes ante la muerte y adherencia terapéutica, del paciente con insuficiencia renal crónica en tratamiento sustitutivo de diálisis peritoneal y su cuidador primario en la unidad de medicina familiar 642012 (tesis de especialidad). Universidad Autónoma del Estado de México, Toluca, México. Recuperado de http:// ri.uaemex.mx/bitstream/handle/20.500.11799/13986/396325.pdf?sequence=1

Hidalgo, C., Ramírez, G., Rojas, J., Hidalgo, A., y Skalicky, A. (2017). Comportamientos de riesgo para la salud y rendimiento académico durante la educación media, según sexo, en estudiantes chilenos. Salud \& Sociedad, 3, 244. Recuperado de http://www. redalyc.org/articulo.oa?id=439754607004

Huitrón, G., Denova, E., Halley, E., Santander, S., Bórquez, M., Zapata, ... Villarroel, L. (2011). Conductas de riesgo en una muestra de adolescentes chilenos y mexicanos: un estudio comparativo. Papeles de Población, 70, 40-42. Recuperado de http:// www.redalyc.org/articulo.oa?id=11221584003

Kübler-Ross, E. (1969). Sobre la muerte y los moribundos. Recuperado de http:// bibliotecaparalapersonaepimeleia.com/greenstone/collect/libros1/index/assoc/ hash07f6.dir/doc.pdf.

Le-Breton, D. (2012) Las conductas de riesgo de los jóvenes. Recuperado de https://www. topia.com.ar/articulos/conductas-riesgo-j\%c3\%b3venes 
Morales, K. (2014). Actitud ante la muerte e intensidad de duelo en adultos mayores con pérdida del cónyuge adscritos a la unidad de medicina familiar número 64 del instituto mexicano del seguro social en el periodo comprendido de marzo a agosto del 2013 (tesis de posgrado). Universidad Autónoma del Estado de México, Toluca, México. Recuperado de http://ri.uaemex.mx/bitstream/handle/20.500.11799/14505/411451. pdf?sequence $=1$

Organización Mundial de la Salud. (2009) Conductas de riesgo en niños y jóvenes. Revista Educativa. Recuperado de http://www.revistaeducativa. com/2018/10\#sdfootnote2anc/19/conductas-de-riesgo-en-ninos-y-jovenes/

Partnership for Droug-Free Kids. (2 de enero del 2017). Las 8 razones principales por las cuales los adolescentes experimentan con drogas y alcohol [mensaje en un blog]. Recuperado de https://drugfree.org/parent-blog/las-8-razones-principales-por-lascuales-los-adolescentes-experimentan-con-drogas-y-alcohol/

Tejedor, D. (2018). 8 motivos: ¿Por qué los jóvenes consumen drogas? Recuperado de https:// caracterurbano.com/psicologia/por-que-jovenes-consumen-droga

Uribe, A., Valderrama, L., y López S. (2007). Actitud y miedo ante la muerte en adultos mayores. Pensamiento Psicológico, 8, 118-120. Recuperado de http://www.redalyc. org/articulo.oa?id=80130809

Wong, P., Reker, G., y Gesser, G. (1994). Death Attitudes Profile-Revised. En R. A. Neimeyer (Ed.), Death Anxiety Handbook: Research, Instrumentation and Application (pp. 121-148). Washington: Taylor \& Francis. 